\title{
From graphs to free products
}

\author{
Madhushree Basu, Vijay Kodiyalam and V.S.Sunder \\ The Institute of Mathematical Sciences, Chennai, India \\ e-mail: madhushree@imsc.res.in, vijay@imsc.res.in, sunder@imsc.res.in
}

November 21, 2018

\begin{abstract}
We investigate a construction which associates a finite von Neumann algebra $M(\Gamma, \mu)$ to a finite weighted graph $(\Gamma, \mu)$. Pleasantly, but not surprisingly, the von Neumann algebra associated to to a 'flower with $n$ petals' is the group von Neumann algebra of the free group on $n$ generators. In general, the algebra $M(\Gamma, \mu)$ is a free product, with amalgamation over a finite-dimensional abelian subalgebra corresponding to the vertex set, of algebras associated to subgraphs 'with one edge' (or actually a pair of dual edges). This also yields 'natural' examples of (i) a Fock-type model of an operator with a free Poisson distribution; and (ii) $\mathbb{C} \oplus \mathbb{C}$-valued circular and semicircular operators.
\end{abstract}

\section{Preliminaries}

There has been a serendipitous convergence of investigations being carried out independently by us on the one hand, and by Guionnet, Jones and Shlyakhtenko on the other - see [GJS1], [KS1], [KS2], [GJS2]. As it has turned out, we have been providing independent proofs, from slightly different viewpoints, of the same facts. Both the papers [KS2] and [GJS2], establish that a certain von Neumann algebra associated to a graph is a free product with amalgamation of a family of von Neumann algebras corresponding to simpler graphs. The amalgamated product involved subgraphs indexed by vertices in [KS2], while the subgraphs are indexed by edges in [GJS2]. This paper was motivated by trying to understand how the proof of our result in [KS2] was also drastically simplfied by considering edges rather than vertices. And, this third episode in our series seems to have the following points in its favour: 
- It does make certain cumulant computations and consequent free independence assertions much more transparent.

- It brings to light a quite simple 'Fock-type model' of free Poisson variables.

- By allowing non-bipartite graphs, we get the aesthetically pleasing fact mentioned in the abstract regarding the 'flower on $n$ petals'.

We investigate, in a little more detail, the construction in [KS2] which associated a von Neumann probability space to a weighted graph. We begin by recalling the set-up:

By a weighted graph we mean a tuple $\Gamma=(V, E, \mu)$, where:

- $V$ is a (finite) set of vertices;

- $E$ is a (finite) set of edges, equipped with 'source' and 'range' maps $s, r: E \rightarrow V$ and '(orientation) reversal' invoution map $E \ni e \mapsto \tilde{e} \in E$ with $(s(e), r(e))=(r(\tilde{e}), s(\tilde{e}))$; and

- $\mu: V \rightarrow(0, \infty)$ is a 'weight or spin function' so normalised that $\sum_{u \in V} \mu^{2}(v)=1$

We let $\mathcal{P}_{n}=\mathcal{P}_{n}(\Gamma)$ denote the set of paths of length $n$ in $\Gamma$ and let $P_{n}(\Gamma)$ denote the vector space with basis $\left\{[\xi]: \xi \in \mathcal{P}_{n}(\Gamma)\right\}$. We think of $\xi=\xi_{1} \xi_{2} \cdots \xi_{n}$ as the 'concatenation product' where $\xi_{i}$ denotes the $i$-th edge of $\xi$. We write $F(\Gamma)=\oplus_{n \geq 0} P_{n}(\Gamma)$ for the indicated direct sum, and equip it with the following slightly complicated multiplication: if $\xi \in \mathcal{P}_{m}(\Gamma), \eta \in \mathcal{P}_{n}(\Gamma)$, then $[\xi] \#[\eta]=$ $\sum_{k=0}^{\min (m, n)}\left[\zeta_{k}\right]$, where $\zeta_{k} \in \mathcal{P}_{m+n-2 k}$ is defined by

$\zeta_{k}= \begin{cases}\frac{\mu\left(v_{m}^{\xi}\right)}{\mu\left(v_{m-k}^{\xi}\right)}\left[\xi_{1} \xi_{2} \cdots \xi_{m-k} \eta_{k+1} \eta_{k+2} \cdots \eta_{n}\right] & \text { if } \xi_{m-j+1}=\widetilde{\eta}_{j} \forall 1 \leq j \leq k \\ 0 & \text { otherwise }\end{cases}$

Here, and elsewhere, we adopt the convention that if $\xi \in \mathcal{P}_{n}$, then $\xi=\xi_{1} \xi_{2} \cdots \xi_{n}$ denotes concatenation product, with $\xi_{i} \in E$ and we write $s\left(\xi_{i}\right)=v_{i-1}^{\xi}$ (so also $\left.r\left(\xi_{i}\right)=s\left(\xi_{i+1}\right)=v_{i}^{\xi}\right)$.

In particular, notice that $\mathcal{P}_{0}(\Gamma)=\{v: v \in V\}$, and that if $v=$ $s(\xi), w=r(\xi)$ for some $\xi \in \mathcal{P}_{n}$, and if $u_{1}, u_{2} \in V$, then $\left[u_{1}\right][\xi]\left[u_{2}\right]=$ $\delta_{u_{1}, v} \delta_{u_{2}, w}[\xi]$; and less trivially, if $\xi \in \mathcal{P}_{1}$ and $\eta \in \mathcal{P}_{m}, m \geq 1$, then

$[\xi] \#[\eta]= \begin{cases}0 & \text { if } r(\xi) \neq s(\eta) \\ {\left[\xi \eta_{1} \ldots \eta_{m}\right]} & \text { if } r(\xi)=s(\eta) \text { but } \xi \neq \widetilde{\eta_{1}} \\ {\left[\xi \eta_{1} \ldots \eta_{m}\right]+\frac{\mu(r(\xi))}{\mu(s(\xi))}\left[\eta_{2} \cdots \eta_{m}\right]} & \text { if } \xi=\widetilde{\eta_{1}}\end{cases}$ 
We define $\phi: F(\Gamma) \rightarrow P_{0}$ by requiring that if $\xi \in P_{n}$, then

$$
\phi([\xi])= \begin{cases}0 & \text { if } n>0 \\ {[\xi]} & \text { if } n=0\end{cases}
$$

and finally define

$$
\tau=\mu^{2} \circ \phi
$$

where we simply write $\mu^{2}$ for the linear extension to $P_{0}(\Gamma)$ which agrees with $\mu^{2}$ on the basis $\mathcal{P}_{0}(\Gamma)$.

It was shown in $[\mathrm{KS}] 1$ that $(F(\Gamma), \tau)$ is a tracial non-commutative *-probability space, with $e^{*}=\tilde{e}$, that the mapping $y \mapsto x y$ extends to a $*$-algebra representation $F(\Gamma) \rightarrow \mathcal{L}\left(L^{2}(F(\Gamma)), \tau\right)$ and that $M(\Gamma, \mu)=\lambda(F \Gamma))^{\prime \prime} \subset \mathcal{L}\left(L^{2}(F(\Gamma)), \tau\right)$ is in standard form. Before proceeding further, it is worth noting that for $\xi, \eta \in \cup_{n} \mathcal{P}_{n}(\Gamma)$, we have

$$
\tau\left([\xi] \#[\eta]^{*}\right)=\delta_{\xi, \eta} \mu(r(\xi)) \mu(s(\xi)),
$$

and hence, if we write $\{\xi\}=(\mu(s(\xi)) \mu(r(\xi)))^{-\frac{1}{2}}[\xi]$, then $\{\{\xi\}: \xi \in$ $\left.\cup_{n \geq 0} \mathcal{P}_{n}(\Gamma)\right\}$ is an orthonormal basis for $\mathcal{H}(\Gamma)=L^{2}(F(\Gamma), \tau)$.

\section{The building blocks}

Our interest here is the examination of just how $M(\Gamma, \mu)$ depends on $(\Gamma, \mu)$. We begin by spelling out some simple examples, which will turn out to be building blocks for the general case.

EXAmple 2.1. 1. Suppose $|V|=|E|=1$, say $V=\{v\}$ and $E=$ $\{e\}$. Then we must have $e=\tilde{e}, s(e)=r(e)=v, \mu(v)=1, \mathcal{P}_{n}=$ $\left\{e^{n}\right\}$ and $\left\{\xi(n)=\left\{e^{n}\right\}: n \geq 0\right\}$ (where $\left\{e^{0}\right\}=\{v\}$ ) is an orthonormal basis for $\mathcal{H}(\Gamma)$; and the definitions show that $x=$ $\lambda(e)$ satisfies $x \xi_{n}=\xi(n+1)+\xi(n-1)$. Thus $x$ is a semi-circular element and $M(\Gamma)=\{x\}^{\prime \prime} \cong L \mathbb{Z}$.

2. Suppose $|V|=1,|E|=2$, say $V=\{v\}$ and $E=\left\{e_{1}, e_{2}\right\}$ suppose $e_{2}=\widetilde{e_{1}}$. Then we must have $s\left(e_{j}\right)=r\left(e_{j}\right)=v, \mu(v)=1$. Further $\left\{\left\{e_{1}\right\},\left\{e_{2}\right\}\right\}$ is an orthonormal basis for $\mathcal{H}_{2}=P_{1}(\Gamma)$, and $P_{n}(\Gamma)$ is isomorphic to $\otimes^{n} \mathcal{H}_{2}$. Thus $\mathcal{H}(\Gamma)$ may be identified with the full Fock space $\mathcal{F}\left(\mathcal{H}_{2}\right)$ and the definitions show that $x_{1}=\lambda\left(e_{1}\right)$ may be identifed as $x_{1}=l_{1}+l_{2}^{*}$, where the $l_{j}$ denote the standard creation operators. It follows that $x_{1}$ is a circular element and $M(\Gamma)=\left\{x_{1}\right\}^{\prime \prime} \cong L F_{2}$.

\footnotetext{
${ }^{1}$ Actually, [KS] treated only the case of bipartite graphs, and sometimes restricted attention to the case of the Perron-Frobenius weighting; but for the the proof of statements made in this paragraph, none of those restrictions is necessary.
} 
3. Suppose $|V|=2,|E|=2$, say $V=\{v, w\}$ and $E=\{e, \widetilde{e}\}$ and suppose $s(e)=v, r(e)=w$ and $\mu(w) \leq \mu(v)$. Write $\rho=$ $\frac{\mu(v)}{\mu(w)}(\geq 1)$. If we let $p_{v}=\lambda([v]), p_{w}=\lambda([w])$, it follows that $\mathcal{H}_{v}=$ ran $p_{v}$ (resp., $\mathcal{H}_{w}=$ ran $p_{w}$ ) has an orthonormal basis given by $\{\{\eta(n)\}: n \geq 0\}$ (resp., $\{\{\xi(n)\}: n \geq 0\}$ where $\eta(n) \in$ $\mathcal{P}_{n}$ (resp., $\left.\xi(n) \in \mathcal{P}_{n}\right)$ and $\eta(n)_{k}=e$ or $\tilde{e}$ (resp., $\xi(n)_{k}=\tilde{e}$ or e according as $k$ is odd or even).

Writing $x=\lambda(e)$, we see that with respect to the decomposition $\mathcal{H}(\Gamma)=\mathcal{H}_{v} \oplus \mathcal{H}_{w}$, the operator $x$ has a matrix decomposition of the form

$$
x=\left[\begin{array}{ll}
0 & t \\
0 & 0
\end{array}\right]
$$

where $t \in \mathcal{L}\left(\mathcal{H}_{w}, \mathcal{H}_{v}\right)$ is seen to be given by

$$
\begin{aligned}
t[\xi(n)] & =x[\xi(n)] \\
& =[e] \#[\tilde{e} e \tilde{e} e \cdots(n \text { terms })] \\
& =[\eta(n+1)]+\rho^{-1}[\eta(n-1)]
\end{aligned}
$$

and hence,

$$
\begin{aligned}
t\{\xi(n)\} & =\left(\mu \left(s(\xi(n)) \mu(r(\xi(n)))^{-\frac{1}{2}} t[\xi(n)]\right.\right. \\
& =\left(\mu(w) \mu(r(\xi(n)))^{-\frac{1}{2}}\left([\eta(n+1)]+\rho^{-1}[\eta(n-1)]\right)\right. \\
& =\left(\rho^{-1} \mu(v) \mu(r(\eta(n \pm 1)))^{-\frac{1}{2}}\left([\eta(n+1)]+\rho^{-1}[\eta(n-1)]\right)\right. \\
& =\rho^{\frac{1}{2}}\{\eta(n+1)\}+\rho^{-\frac{1}{2}}\{\eta(n-1)\}
\end{aligned}
$$

It is a fact - see Proposition 2.2 - that $t^{*} t$ has has absolutely continuous spectrum. This fact has two consequences:

(i) if $t=u|t|$ is the polar decomposition of $t$, then $u$ maps $\mathcal{H}_{w}$ isometrically onto the subspace $\mathcal{M}=\overline{\text { ran } t}$ of $\mathcal{H}_{v}$, and if $z$ is the projection onto $\mathcal{H}_{v} \ominus \mathcal{M}$ then $\tau(z)=\mu^{2}(v)-\mu^{2}(w)$; and

(ii) $W^{*}(|t|) \cong L \mathbb{Z}$.

Since $p_{v}+p_{w}=1$ and $z \leq p_{v}$, the definitions are seen to show that $M(\Gamma, \mu)$ is isomorphic to $\mathbb{C} \oplus M_{2}(L \mathbb{Z})$ via the unique isomorphism which maps $p_{v}, p_{w}, z, u$ and $|t|$, respectively, to $\left(1,\left(\begin{array}{ll}1 & 0 \\ 0 & 0\end{array}\right)\right),\left(0,\left(\begin{array}{ll}0 & 0 \\ 0 & 1\end{array}\right)\right),\left(1,\left(\begin{array}{ll}0 & 0 \\ 0 & 0\end{array}\right)\right),\left(0,\left(\begin{array}{ll}0 & 1 \\ 0 & 0\end{array}\right)\right)$, and $\left(1,\left(\begin{array}{ll}0 & 0 \\ 0 & a\end{array}\right)\right)$ for some positive a with absolutely continuous spectrum which generates $L \mathbb{Z}$ as a von Neumann algebra. (This 
must be compared with Lemma 17 of [GJS2], bearing in mind that their $\mu$ is our $\mu^{2}$.)

Proposition 2.2. Let $\ell^{2}(\mathbb{N})$ have its standard orthonormal basis $\left\{\delta_{n}: n \in \mathbb{N}\right\}$. (For us, $\mathbb{N}=\{0,1,2, \cdots\}$.) Let $\ell \delta_{n}=\delta_{n+1}$ denote the creation operator (or unilateral shift), with $\ell^{*} \delta_{n}=\delta_{n-1}$ (where $\left.\delta_{-1}=0\right)$. Let $\rho>1$ and $t=\rho^{\frac{1}{2}} \ell+\rho^{-\frac{1}{2}} \ell^{*}$. Then,

1. $t^{*} t$ leaves the subspace $\ell^{2}(2 \mathbb{N})$ invariant;

2. $\delta_{0}$ is a cyclic vector for the restriction to $\ell^{2}(2 \mathbb{N})$ of $t^{*} t$, call it $a_{\rho} ;$ and

3. the (scalar) spectral measure of $a_{\rho}$ associated to $\delta_{0}$ is absolutely continuous with respect to Lebesgue measure; in fact $a_{\rho}$ has a free Poisson distribution.

Proof. A little algebra shows that

$$
\begin{aligned}
t^{*} t & =\left(\rho^{\frac{1}{2}} \ell^{*}+\rho^{-\frac{1}{2}} \ell\right)\left(\rho^{\frac{1}{2}} \ell+\rho^{-\frac{1}{2}} \ell^{*}\right) \\
& =\ell^{2}+\ell^{* 2}+\left(\rho+\rho^{-1}\right)-\rho^{-1} p_{0},
\end{aligned}
$$

where $p_{0}$ is the rank one projection onto $\mathbb{C} \delta_{0}$. It is seen that this operator leaves both subspaces $\ell^{2}(2 \mathbb{N})$ and $\ell^{2}(2 \mathbb{N}+1)$ invariant, with its restrictions to these subspaces being unitarily equivalent to $\ell+$ $\ell^{*}+\left(\rho+\rho^{-1}\right)-\rho^{-1} p_{0}$ and $\ell+\ell^{*}$ respectively. Since the spectral type does not change under scalar translation, we may assume without loss of generality that $a_{\rho}=\ell+\ell^{*}-\rho^{-1} p_{0}$ and establish that $a_{0}$ has absolutely continuous scalar spectral measure corresponding to $\delta_{0}$.

Write $a_{0}=\ell+\ell^{*}$ so that $a_{\rho}=a_{0}-\rho^{-1} p_{0}$. Let the scalar spectral measures of $a_{0}$ and $a_{\rho}$ be denoted by $\mu$ and $\mu_{\rho}$ respectively, and consider their Cauchy transforms given by

$$
F_{\lambda}(z)=\left\langle\left(a_{\lambda}-z\right)^{-1} \delta_{0}, \delta_{0}\right\rangle=\int_{\mathbb{R}} \frac{d \mu_{\lambda}(x)}{x-z}
$$

for $\lambda \in\{0, \rho\}$ and $z \in \mathbb{C}^{+}=\{\zeta \in \mathbb{C}: \operatorname{Im}(\zeta)>0\}$.

It follows from the resolvent equation that

$$
\begin{aligned}
F_{\rho}(z) & =\left\langle\left(a_{\rho}-z\right)^{-1} \delta_{0}, \delta_{0}\right\rangle \\
& =\left\langle\left(a_{0}-z\right)^{-1} \delta_{0}, \delta_{0}\right\rangle+\left\langle\left(a_{\rho}-z\right)^{-1} \rho^{-1} p_{0}\left(a_{\lambda}-z\right)^{-1} \delta_{0}, \delta_{0}\right\rangle \\
& =F_{0}(z)+\rho^{-1} F_{\rho}(z) F_{0}(z) ;
\end{aligned}
$$

Hence

$$
F_{\rho}(z)=\frac{F_{0}(z)}{1-\rho^{-1} F_{0}(z)}=\frac{\rho F_{0}(z)}{\rho-F_{0}(z)}
$$


It is seen from Lemma 2.21 of [NS] - after noting that the $G$ of that Lemma is the negative of the $F_{0}$ here - that $F_{0}(z)=\frac{z-\sqrt{z^{2}-4}}{2}$ where $\sqrt{z^{2}-4}$ is a branch of that square root such that $\sqrt{z^{2}-4}=$ $\sqrt{z+2} \sqrt{z-2}$ where the two individual factors are respectively defined by using the branch-cuts $\{\mp 2-i t: t \in(0, \infty)$. (This choice ensures that $\lim _{|z| \rightarrow \infty} F_{0}(z)=0$, which is clearly necessary.) It follows that $F_{0}$, which is holomorphic in $\mathbb{C}^{+}$, actually extends to a continuous function on $\mathbb{C}^{+} \cup \mathbb{R}$, and that if we write $f_{0}(a)=\lim _{b \downarrow 0} F_{0}(a+i b)$, then we have

$$
2 f_{0}(t)= \begin{cases}-t+\sqrt{t^{2}-4} & \text { if } t \geq 2 \\ -t+i \sqrt{4-t^{2}} & \text { if } t \in[-2,2] \\ -t-\sqrt{t^{2}-4} & \text { if } t \leq-2\end{cases}
$$

It is easy to check that $f_{0}$ is strictly increasing in $(-\infty,-2)$, as well as in in $(2, \infty)$, has non-zero imaginary part in $(-2,2)$, and satisfies $f(\mathbb{R} \backslash(-2,2))=[-1,0) \cup(0,1]$. Since $\rho>1$, we may deduce that $F_{0}(z) \neq \rho \forall z \in \mathbb{C}^{+} \cup \mathbb{R}$, and hence that also $F_{\rho}$ extends to a continuous function on $\mathbb{C}^{+} \cup \mathbb{R}$ with equation (2.1) continuing to hold for all $z \in \mathbb{C}^{+} \cup \mathbb{R}$. Writing $f_{\lambda}(t)=F_{\lambda}(t+i 0)$ for $\lambda \in\{0, \rho\}$, we find that

$$
f_{\rho}(t)=\frac{\rho f_{0}(t)}{\rho-f_{0}(t)}=\frac{1}{f_{0}(t)^{-1}-\rho^{-1}}
$$

and hence that

$$
\begin{aligned}
\operatorname{Im}\left(f_{\rho}(t)\right) & =-\frac{\operatorname{Im}\left(f_{0}(t)^{-1}\right)}{\left|f_{0}(t)^{-1}-\rho^{-1}\right|^{2}} \\
& =\frac{\operatorname{Im}\left(f_{0}(t)\right)}{\left|1-f_{0}(t) \rho^{-1}\right|^{2}} \\
& =\rho^{2} \frac{\operatorname{Im}\left(f_{0}(t)\right)}{\left|f_{0}(t)-\rho\right|^{2}} \\
& =1_{[-2,2]}(t) \frac{\rho^{2} \sqrt{4-t^{2}}}{2\left|f_{0}(t)-\rho\right|^{2}} .
\end{aligned}
$$

Now, for $t \in[-2,2]$, we see that

$$
\begin{aligned}
\left|f_{0}(t)-\rho\right|^{2} & =\left|\frac{-t+i \sqrt{4-t^{2}}}{2}-\rho\right|^{2} \\
& =\frac{1}{4}\left((t+2 \rho)^{2}+4-t^{2}\right) \\
& =\rho^{2}+\rho t+1 .
\end{aligned}
$$

It follows from Stieltje's inversion formula that our $a_{\rho}$ has absolutely continuous scalar spectral measure $\mu_{\rho}$, with density given 
by

$$
\begin{aligned}
g_{\rho}(t) & =\frac{1}{\pi} \operatorname{Im} f_{\rho}(t) \\
& =1_{[-2,2]}(t) \frac{\rho^{2} \sqrt{4-t^{2}}}{2 \pi\left(\rho^{2}+\rho t+1\right)} .
\end{aligned}
$$

Hence the operator $t^{*} t=a_{\rho}+\left(\rho+\rho^{-1}\right) 1$ has has absolutely continuous scalar spectral measure, with density given by

$$
\begin{aligned}
g(t) & =g_{\rho}\left(t-\left(\rho+\rho^{-1}\right)\right) \\
& =1_{\left[\left(\rho+\rho^{-1}\right)-2,\left(\rho+\rho^{-1}\right)+2\right]}(t) \frac{\rho^{2}{\sqrt{4-\left(t-\left(\rho+\rho^{-1}\right)\right.}}^{2}}{2 \pi \rho^{-2}\left(\rho^{2}+\rho\left(t-\rho-\rho^{-1}\right)+1\right)} \\
& =1_{\left[\left(\rho+\rho^{-1}\right)-2,\left(\rho+\rho^{-1}\right)+2\right]}(t) \frac{\rho^{2}{\sqrt{4-\left(t-\left(\rho+\rho^{-1}\right)\right.}}^{2}}{2 \pi \rho^{-1} t}
\end{aligned}
$$

If we write $\lambda=\rho^{2}$ and $\alpha=\rho^{-1}$, we see that $\alpha(1+\lambda)$ and recognise the fact that not only does $t^{*} t$ have absolutely continuous spectrum, but - by comparing with equation (12.15) of [NS] -even that it actually has a free Poisson distribution, with rate $\rho^{2}$ and jump size $\rho^{-1}$. However, we actually discovered this fact about $t^{*} t$ having a free Poisson distribution with the stated $\lambda$ and $\alpha$ by a cute cumulant computation which we present in the final section, both for giving a combinatorial rather than analytic proof of this Proposition, and because we came across that proof first.

\section{Some free cumulants}

Before proceeding with the further study of a general $(\Gamma, \mu)$, we will need an alternative description of $M(\Gamma, \tau)$.

Let $\operatorname{Gr}(\Gamma)=\oplus_{n \geq 0} P_{n}(\Gamma)$ be equipped with a $*$-algebra structure wherein $[\xi] \circ[\eta]=[\xi \eta])$ and $[\xi]^{*}=[\tilde{\xi}]=\left[\widetilde{\xi_{n}} \cdots \widetilde{\xi_{1}}\right]$ for $\xi \in \mathcal{P}_{n}, \eta \in \mathcal{P}_{m}$. It turns out - see $[\mathrm{KS} 2$ - that $\operatorname{Gr}(\Gamma)$ and $F(\Gamma)$ are isomorphic as *-algebras. While the multiplication is simpler in $\operatorname{Gr}(\Gamma)$, the trace $\tau$ on $F(\Gamma)$ turns out, when transported by the above isomorphism, to be given by a slightly more complicated formula. (It is what has been called the Voiculescu trace by Jones et al.) We shall write $t r$ for this transported trace on $G r(\Gamma)$, and $\phi$ for the $t r$-preserving conditional expectation of $M(\Gamma, \mu)\left(=\lambda(G r(\Gamma))^{\prime \prime}\right)$ onto $P_{0}(\Gamma)$. We shall use the

\footnotetext{
${ }^{2}$ The remark made in an earlier footnote, concerning assumptions regarding bipartiteness of $\Gamma$, applies here as well.
} 
same letter $\phi$ to denote restrictions to subalgebras which contain $P_{0}(\Gamma)$.

We wish to regard $(\operatorname{Gr}(\Gamma), \phi)$ as an operator-valued non-commutative probability space over $P_{0}(\Gamma)$, our first order of business being the determination of the $P_{0}(\Gamma)$-valued mixed cumulants in $\operatorname{Gr}(\Gamma)$.

Proposition 3.1. The $P_{0}(\Gamma)$-valued mixed cumulants in $G r(\Gamma)$ are given thus:

$\kappa_{n}\left(\left[e_{1}\right],\left[e_{2}\right], \cdots,\left[e_{n}\right]\right)=0$ unless $n=2$ and $e_{2}=\widetilde{e_{1}}$; and if $e_{2}=$ $\widetilde{e_{1}}$ with $s\left(e_{1}\right)=v, r\left(e_{1}\right)=w$, then $\kappa_{2}\left(\left[e_{1}\right],\left[\widetilde{e_{1}}\right]\right)=\frac{\mu(w)}{\mu(v)}[v]$.

Proof. The proof depends on the 'moment-cumulant' relations which guarantee that in order to prove this proposition, it will suffice to establish the following, which is what we shall do:

(a) Define $\kappa_{n}:(G r(\Gamma))^{n} \rightarrow P_{0}(\Gamma)$ to be the unique multilinear map which is defined when the arguments are tuples of paths as asserted in the proposition; note that (i) it is 'balanced' over $P_{0}(\Gamma)$ in the sense that $\kappa_{n}\left(x_{1}, \cdots, x_{i-1} b, x_{i}, \cdots, x_{n}\right)=\kappa_{n}\left(x_{1}, \cdots, x_{i-1}, b x_{i}, \cdots, x_{n}\right)$ for all $x_{j} \in G r(\Gamma), b \in P_{0}(\Gamma)$ and $1<i \leq n$, and (ii) is $P_{0}(\Gamma)$-bilinear meaning $\kappa_{n}\left(b x_{1}, x_{2}, \cdots, x_{n-1}, x_{n} b^{\prime}\right)=b \kappa_{n}\left(x_{1}, x_{2}, \cdots, x_{n-1}, x_{n}\right) b^{\prime}$ for all $x_{j} \in G r(\Gamma), b, b^{\prime} \in P_{0}(\Gamma)$;

(b) define the 'multiplicative extensions' $\kappa_{\pi}:(G r(\Gamma))^{n} \rightarrow P_{0}(\Gamma)$ for $\pi \in N C(n)$ by requiring, inductively, that if $[k, l]$ is an interval constituting a class of $\pi$, and if we write $\sigma$ for the element of $N C(n-$ $l+k-1)$ given by the restriction of $\pi$ to $\{1, \cdots, k-1, l+1, \cdots, n\}$, so that ' $\pi=\sigma \bigvee 1_{[k, l]}$ ' then

$$
\begin{aligned}
\kappa_{\pi}\left(x_{1}, \cdots, x_{n}\right) & =\kappa_{\sigma}\left(x_{1}, \cdots, x_{k-1} \kappa_{l-k+1}\left(x_{k}, \cdots, x_{l}\right), x_{l+1}, \cdots, x_{n}\right) \\
& =\kappa_{\sigma}\left(x_{1}, \cdots, x_{k-1}, \kappa_{l-k+1}\left(x_{k}, \cdots, x_{l}\right) x_{l+1}, \cdots, x_{n}\right) ;
\end{aligned}
$$

(c) and verify that for any $e_{1}, \cdots, e_{n} \in \mathcal{P}_{1}(\Gamma)$,

$$
\phi\left(\left[e_{1}\right] \cdots\left[e_{n}\right]\right)=\sum_{\pi \in N C(n)} \kappa_{\pi}\left(\left[e_{1}\right],\left[e_{2}\right], \cdots,\left[e_{n}\right]\right) .
$$

For this verification, we first assert that if $e_{1}, e_{2}, \cdots, e_{n} \in E$ and $\pi \in N C(n)$, the quantity $\kappa_{\pi}\left(\left[e_{1}\right],\left[e_{2}\right], \cdots,\left[e_{n}\right]\right)$ (yielded by the unique 'multiplicative extension' of the $\kappa_{n}$ 's as in (b) above) can be non-zero only if

(i) $e_{1} e_{2} \cdots e_{n}$ is a meaningfully defined loop based at $s\left(e_{1}\right)$, meaning $f\left(e_{i}\right)=s\left(e_{i+1}\right)$ for $1 \leq i \leq n$, with $e_{n+1}$ being interpreted as $e_{1}$;

(ii) $\pi \in N C_{2}(n)$ is a pair partition of $n$ (and in particular $n$ is even), such that $\{i, j\} \in \pi \Rightarrow e_{j}=\widetilde{e_{i}}$; 
and if that is the case, then,

$$
\kappa_{\pi}\left(\left[e_{1}\right],\left[e_{2}\right], \cdots,\left[e_{n}\right]\right)=\left(\prod_{\substack{\{i, j\} \in \pi \\ i<j}} \frac{\mu\left(r\left(e_{i}\right)\right.}{\mu\left(r\left(e_{j}\right)\right.}\right)\left[s\left(e_{1}\right)\right] .
$$

We prove this assertion by induction on $n$. This is trivial for $n=1$ since $\kappa_{1} \equiv 0$. By the inductive definition of the multiplicative extension, it is clear that if $\kappa_{\pi}\left(\left[e_{1}\right],\left[e_{2}\right], \cdots,\left[e_{n}\right]\right)$ is to be non-zero, $\pi$ must contain an interval class of the form $\{k, k+1\}$ such that $e_{k+1}=\widetilde{e_{k}}$; if $\sigma$ denotes $\left.\pi\right|_{\{1,2, \cdots, k-1, k+2, \cdots n\}}$ we must have

$$
\begin{aligned}
\kappa_{\pi}\left(\left[e_{1}\right], \cdots,\left[e_{n}\right]\right) & =\frac{\mu\left(r\left(e_{k}\right)\right)}{\mu\left(r\left(e_{k+1}\right)\right)} \kappa_{\sigma}\left(\left[e_{1}\right], \cdots,\left[e_{k-1}\right]\left[s\left(e_{k}\right)\right],\left[e_{k+2}\right], \cdots,\left[e_{n}\right]\right) \\
& =\frac{\mu\left(r\left(e_{k}\right)\right)}{\mu\left(r\left(e_{k+1}\right)\right)} \kappa_{\sigma}\left(\left[e_{1}\right], \cdots,\left[e_{k-1}\right],\left[s\left(e_{k}\right)\right]\left[e_{k+2}\right], \cdots,\left[e_{n}\right]\right) \\
& =\frac{\mu\left(r\left(e_{k}\right)\right)}{\mu\left(r\left(e_{k+1}\right)\right)} \kappa_{\sigma}\left(\left[e_{1}\right], \cdots,\left[e_{k-1}\right]\left[r\left(e_{k+1}\right)\right],\left[e_{k+2}\right], \cdots,\left[e_{n}\right]\right) ;
\end{aligned}
$$

and for this to be non-zero, we must have $r\left(e_{k-1}\right)=s\left(e_{k}\right)=r\left(e_{k+1}\right)=$ $s\left(e_{k+2}\right)$, in which case we would have

$$
\kappa_{\pi}\left(\left[e_{1}\right], \cdots,\left[e_{n}\right]\right)=\frac{\mu\left(r\left(e_{k}\right)\right)}{\mu\left(r\left(e_{k+1}\right)\right)} \kappa_{\sigma}\left(\left[e_{1}\right], \cdots,\left[e_{k-1}\right],\left[e_{k+2}\right], \cdots,\left[e_{n}\right]\right),
$$

and the requirement that $\kappa_{\sigma}\left(\left[e_{1}\right], \cdots,\left[e_{k-1}\right],\left[e_{k+2}\right], \cdots,\left[e_{n}\right]\right)$ be nonzero, along with the induction hypothesis, finally completes the proof of the assertion.

Now, in order to verify equation 3.3 , it suffices to check that for any $v \in V$, we have

$$
\operatorname{tr}\left(\left[e_{1}\right]\left[e_{2}\right] \cdots\left[e_{n}\right][v]\right)=\sum_{\pi \in N C(n)} \operatorname{tr}\left(\kappa_{\pi}\left(\left[e_{1}\right],\left[e_{2}\right], \cdots,\left[e_{n}\right]\right)[v]\right) .
$$

First observe that both sides of equation 3.5 vanish unless $e_{1} \cdots e_{n}$ is a meaningfully defined path with both source and range equal to $v$ (since $t r$ is a trace and $[v]$ is idempotent). In view of our description above of the multiplicative extension $\kappa_{\pi}$, we need, thus, to verify that for such a loop, we have

$$
\operatorname{tr}\left(\left[e_{1} \cdots e_{n}\right]\right)=\sum_{\pi \in N C_{2}(n)}\left(\prod_{\substack{\{i, j\} \in \pi \\ i<j}} \delta_{e_{j}, \widetilde{e_{i}}} \frac{\mu\left(r\left(e_{i}\right)\right.}{\mu\left(r\left(e_{j}\right)\right.}\right) \mu^{2}\left(s\left(e_{1}\right)\right),
$$


but that is indeed the case (see equation (3) and the proof of Proposition 5 in $[\mathrm{KS} 1]$ ).

In order to derive the true import of Proposition 3.1, we should first introduce some notation:

For each dual pair $e, \tilde{e}$ of edges - with, say, $s(e)=v, r(e)=w$ - we shall write $\Gamma_{e}=\left(V_{e}, E_{e}, \mu_{e}\right)$ where $V_{e}=V, \mu_{e}=\mu$ and $E_{e}=\{e, \tilde{e}\}$ (with source, range and reversal in $E_{e}$ as in $E$ ). If $e=\tilde{e}$, the above definitions are to be suitably interpreted. Now for "the true import of Proposition 3.1]:

COROLlary 3.2. With the foregoing notation, we have:

$$
G r(\Gamma, \mu)=*_{P_{0}(\Gamma)}\left\{G r\left(\Gamma_{e}, \mu_{e}\right):\{e, \tilde{e}\} \subset E\right\}
$$

and hence, also

$$
M(\Gamma, \mu)=*_{P_{0}(\Gamma)}\left\{M\left(\Gamma_{e}, \mu_{e}\right):\{e, \tilde{e}\} \subset E\right\}
$$

Proof. Proposition 3.3.3 of [S1] shows that if $A \stackrel{\phi}{\rightarrow} B$ is a "noncommutative probability space over $B$ ', if $\left\{A_{i}: i \in I\right\}$ is a family of subalgebras of $A$ containing $B$, such that $\left\{A_{i}: i \in I\right\}$ generates $A$, and if $G_{i}$ is a set of generators of the algebra $A_{i}$, then $A$ is the free product with amalgamation over $B$ of $\left\{A_{i}: i \in I\right\}$ if and only if the mixed $B$-valued cumulants $\kappa_{n}\left(x_{1}, \cdots, x_{n}\right)$ vanish whenever $x_{1}, \cdots, \cdots x_{n} \in \cup_{i} G_{i}$, unless all the $x_{i}$ belong to the same $G_{k}$ for some $k$. The desired assertion then follows from Proposition 3.1,

The following assertion, advertised in the abstract, is an immediate consequence of Corollary 3.2 and Examples 2.1 (1) and (2).

COROllary 3.3. If $\Gamma_{n}$ denotes the 'flower with $n$ petals' (thus $|V|=$ $1,|E|=n)$, then $M(\Gamma) \cong L \mathbb{F}_{n}$, independent of the reversal map on E.

REMARK 3.4. We may deduce from Proposition 3.1 that the $x=\lambda(e)$ of Example 2.1 (3) is a $P_{0}(\Gamma)$-valued circular operator, in the sense of $[$ Dyk] (see Definition 4.1), with covariance $(\alpha, \beta)$ where $\alpha(b)=$ $\phi\left(x^{*} b x\right)$ and $\beta(b)=\phi\left(x b x^{*}\right)$ for all $b \in \mathcal{P}_{0}$ are the completely positive self-maps of $P_{0}(\Gamma)\left(=\mathbb{C} p_{v} \oplus \mathbb{C} p_{w}\right)$ induced by the matrices

$$
\alpha=\left[\begin{array}{cc}
0 & \rho^{-1} \\
0 & 0
\end{array}\right] \text { and } \beta=\left[\begin{array}{ll}
0 & 0 \\
\rho & 0
\end{array}\right] .
$$


If $s=x+x^{*}$, it follows then that $s$ is a $P_{0}(\Gamma)$-valued semicircular element ( since $\kappa_{n}\left(s b_{1}, s b_{2}, \cdots s b_{n-1}, s\right)=0$ unless $n=2$ and $\kappa_{2}(s b, s)=\eta(b)$ where $\eta$ is the (completely) positive self-map of $\mathbb{C} \oplus \mathbb{C}$ induced by the matrix

$$
\eta=\left[\begin{array}{cc}
0 & \rho^{-1} \\
\rho & 0
\end{array}\right]
$$

\section{Narayana numbers}

Recall the Narayana numbers $N(n, k)$ defined for all $n, k \in \mathbb{N}$ with $1 \leq k \leq n$ by

$$
N(n, k)=|\{\pi \in N C(n):|\pi|=k\}| .
$$

Define the associated polynomials $N_{n}$ by

$$
N_{n}(T)=\sum_{k=1}^{n} N(n, k) T^{k} .
$$

Recall also that a random variable in a non-commutative probability space $(A, \tau)$ is said to be free Poisson with rate $\lambda$ and jump size $\alpha$ if its free cumulants are given by $\kappa_{n}=\lambda \alpha^{n}$ for all $n \in \mathbb{N}$. An easy application of the moment-cumulant relations shows that an equivalent condition for a random variable to be free Poisson with rate $\lambda$ and jump size $\alpha$ is that its moments are given by $\mu_{n}=\alpha^{n} N_{n}(\lambda)$ for all $n \in \mathbb{N}$.

We now illustrate an application of this characterisation of a free Poisson variable in the situation of $\S 2$, Example 2.1 (3). There, $x=\lambda(e)$ has a matrix decomposition involving $t \in \mathcal{L}\left(\mathcal{H}_{w}, \mathcal{H}_{v}\right)$ where $t^{*} t$ was shown to have a free Poisson distribution. We will verify below by a cumulant computation that $t^{*} t$ is free Poisson with rate $\rho^{2}$ and jump size $\rho^{-1}$ in the non-commutative probability space $p_{w} M(\Gamma, \mu) p_{w}$.

Begin by observing that $x^{*} x$ has a non-zero entry only in the $w$ corner and that this entry is $t^{*} t$. Thus the trace in $M(\Gamma, \mu)$ of $\left(x^{*} x\right)^{n}$ is $\mu^{2}(w)$ times the trace - call it $t r_{w}$ - in $p_{w} M(\Gamma, \mu) p_{w}$ of $\left(t^{*} t\right)^{n}$. We now compute $\operatorname{tr}\left(\left(x^{*} x\right)^{n}\right)=\operatorname{tr}\left(\left([e]^{*}[e]\right)^{n}\right)$.

First apply the moment-cumulant relations and Proposition 3.1 to conclude that

$$
\phi\left(\left([e]^{*}[e]\right)^{n}\right)=\sum_{\pi \in N C(2 n)} \kappa_{\pi}\left([e]^{*},[e], \cdots,[e]^{*},[e]\right) .
$$


While this sum ranges over all $\pi \in N C(2 n)$, Proposition 3.1 enables us to conclude that unless $\pi$ is a non-crossing pair partition, its contribution vanishes. Thus we have:

$$
\phi\left(\left([e]^{*}[e]\right)^{n}\right)=\sum_{\pi \in N C_{2}(2 n)} \kappa_{\pi}\left([e]^{*},[e], \cdots,[e]^{*},[e]\right)
$$

Now we use the well-known bijection between non-crossing pair partitions (or equivalently, Temperley-Lieb diagrams) on $2 n$ points and all non-crossing partitions on $n$ points. We will denote this bijection as $\pi \in N C_{2}(2 n) \leftrightarrow \tilde{\pi} \in N C(n)$. This is illustrated by example in Figure 4 for $\pi=\{\{1,8\},\{2,5\},\{3,4\},\{6,7\},\{9,12\},\{10,11\}\}$ and may be summarised by saying that the black regions of the Temperley-Lieb diagram for $\pi \in N C_{2}(2 n)$ correspond to the classes of $\tilde{\pi} \in N C(n)$. Note that in Figure 4 the numbers above refer to the

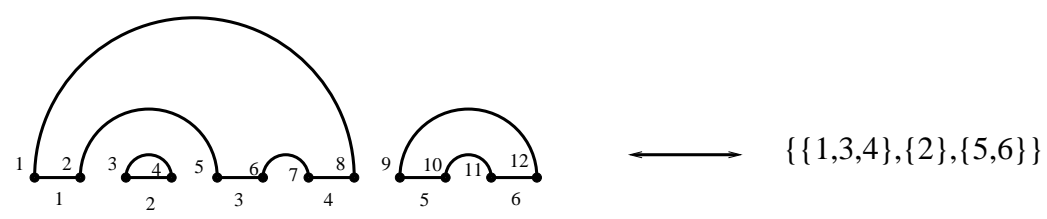

Figure 1: $\pi \in N C_{2}(12) \leftrightarrow \tilde{\pi} \in N C(6)$

vertices while those below refer to the black segments.

It follows from Proposition 3.1 that for any $\pi \in N C_{2}(2 n)$, the term $\kappa_{\pi}\left([e]^{*},[e], \cdots,[e]^{*},[e]\right)$ is a scalar multiple of $p_{w}$ where the scalar is given by a product of $n$ terms each of which is $\rho=\frac{\mu(v)}{\mu(w)}$ or $\rho^{-1}=\frac{\mu(w)}{\mu(v)}$. Classes of $\pi$ for which the smaller element is odd give $\rho$, while those for which the smaller element is even give $\rho^{-1}$. Thus $\kappa_{\pi}\left([e]^{*},[e], \cdots,[e]^{*},[e]\right)$ evaluates to $\rho^{\left(|\pi|_{\text {odd }}-|\pi|_{\text {even }}\right)} p_{w}=\rho^{\left(2|\pi|_{\text {odd }}-n\right)} p_{w}$, where, of course, $|\pi|_{\text {odd }}$ (resp. $|\pi|_{\text {even }}$ ) denotes the number of classes of $\pi$ whose smaller element is odd (resp. even).

Our main combinatorial observation is contained in the following simple lemma.

Lemma 4.1. For any $\pi \in N C_{2}(2 n),|\pi|_{\text {odd }}=|\tilde{\pi}|$.

Proof. We induce on $n$ with the basis case $n=1$ having only one $\pi$ with $|\pi|_{\text {odd }}=|\tilde{\pi}|=1$. For larger $n$, consider a class of $\pi$ of the form $\{i, i+1\}$, and remove it to get $\rho \in N C_{2}(2 n-2)$. A moment's thought shows that if $i$ is odd then $|\pi|_{\text {odd }}=|\rho|_{\text {odd }}+1=|\tilde{\rho}|+1=|\tilde{\pi}|$, while if $i$ is even then $|\pi|_{\text {odd }}=|\rho|_{\text {odd }}=|\tilde{\rho}|=|\tilde{\pi}|$. 
Thus:

$$
\begin{aligned}
\phi\left(\left([e]^{*}[e]\right)^{n}\right) & =\sum_{\pi \in N C_{2}(2 n)} \rho^{\left(2|\pi|_{\text {odd }}-n\right)} p_{w} \\
& =\sum_{\tilde{\pi} \in N C(n)} \rho^{(2|\tilde{\pi}|-n)} p_{w} \\
& =\sum_{k=1}^{n} \sum_{\{\tilde{\pi} \in N C(n):|\tilde{\pi}|=k\}} \rho^{2 k-n} p_{w} \\
& =\sum_{k=1}^{n} N(n, k) \rho^{2 k-n} p_{w}
\end{aligned}
$$

Hence $\operatorname{tr}\left(\left([e]^{*}[e]\right)^{n}\right)=\sum_{k=1}^{n} N(n, k) \rho^{2 k-n} \mu^{2}(w)$ and thus $\operatorname{tr}_{w}\left(\left(t^{*} t\right)^{n}\right)=$ $\sum_{k=1}^{n} N(n, k) \rho^{2 k-n}$. Now the characterisation of free Poisson elements in terms of their moments shows that $t^{*} t$ is free Poisson with rate $\rho^{2}$ and jump size $\rho^{-1}$.

REMARK 4.2. 1. Thus, for $t=\rho^{\frac{1}{2}} \ell+\rho^{-\frac{1}{2}} \ell^{*}$, we have shown that $t^{*} t$ is a free Poisson element with rate $\rho^{2}$ and jump size $\rho^{-1}$. By scaling by an appropriate constant, we can similarly obtain such simple Fock-type models of free Poisson elements with arbitrary jump size and rate.

2. Similar scaling, and the fact that $e^{i \theta} \ell$ is unitarily equivalent to $\ell$ (by a unitary operator which fixes $\delta_{0}$ ) show that, in fact, if $t=a \ell+b \ell^{*}$ for any $a, b \in \mathbb{C}$, then $t^{*} t$ is a free Poisson element.

Acknowledgement: We would like to thank M. Krishna for patiently leading us through the computation of Cauchy transforms of rank-one perturbations as we struggled with an apparent contradiction, which was finally resolved when we realised a problematic minus sign stemming from a small mistake in choice of square roots. (We claim no originality for this problem, for the same incorrect sign also surfaces on page 33 of [NS] - cf. our formula (2.2) and the formula there for $g$, when $-\infty<t<-2$.)

\section{References}

[1] [Dyk] Ken Dykema, Hyperinvariant subspaces for some Bcircular operators, With an appendix by Gabriel Tucci. Math. Ann. 333 (2005), no. 3, 485-523. 
[GJS1] A. Guionnet, V. F. R. Jones and D. Shlayakhtenko, Random matrices, free probability, planar algebras and subfactors, arXiv:0712.2904v2.

[GJS2] A. Guionnet, V.F.R. Jones, D. Shlyakhtenko, A semifinite algebra associated to a planar algebra, e-print arXiv (math.OA)0911.4728.

[KS1] Vijay Kodiyalam and V.S. Sunder, Guionnet-JonesShlyakhtenko subfactors associated to finite-dimensional Kaç algebras, e-print arXiv (math. OA) 09013180.

[KS2] Vijay Kodiyalam and V.S. Sunder, On the Guionnet-JonesShlyakhtenko construction for graphs, e-print arXiv (math. OA) 0911.2047

[NS] A. Nica and R. Speicher, Lectures on the Combinatorics of Free Probability, LMS Lecture Note Series, vol. 335, Cambridge Universities Press, Cambridge, 2006.

[S1] R. Speicher, Combinatorial theory of the free product with amalgamation and operator-valued free probability theory, Memoirs of the AMS 132 (1998).

[S2] R. Speicher, A New Example of 'Independence' and 'White Noise', Probab. Th. Rel. Fields, 84, 141-159 (1990) 